In Situ

Revue des patrimoines
In Situ

Revue des patrimoines

$44 \mid 2021$

Les patrimoines des lycées français du XIXe au XXIe siècle : de la connaissance à la valorisation

\title{
L'architecture des lycées de Metz (1803-2020)
}

High school architecture in the city of Metz (1803-2020)

\section{Pierre Maurer}

\section{(2) OpenEdition}

Journals

Édition électronique

URL : https://journals.openedition.org/insitu/31654

DOI : 10.4000/insitu.31654

ISSN : 1630-7305

Éditeur

Ministère de la Culture

Référence électronique

Pierre Maurer, «L'architecture des lycées de Metz (1803-2020) », In Situ [En ligne], 44 | 2021, mis en ligne le 30 avril 2021, consulté le 14 décembre 2022. URL : http://journals.openedition.org/insitu/

31654 ; DOI : https://doi.org/10.4000/insitu.31654

Ce document a été généré automatiquement le 14 décembre 2022.

\section{(c) (i) $(9)$}

Creative Commons - Attribution - Pas d'Utilisation Commerciale - Pas de Modification 4.0 International - CC BY-NC-ND 4.0

https://creativecommons.org/licenses/by-nc-nd/4.0/ 


\title{
L'architecture des lycées de Metz (1803-2020)
}

\author{
High school architecture in the city of Metz (1803-2020)
}

Pierre Maurer

1 Chef-lieu du département de la Moselle, Metz compte actuellement une dizaine de lycées sur son territoire communal: Fabert, Georges-de-La-Tour, Alain-Fournier, la Communication, Louis-de-Cormontaigne, Louis-Vincent, René-Cassin, Robert-Schuman ou encore le lycée hôtelier Raymond-Mondon, ainsi que des établissements privés (l'Institution de La Salle ou encore l'ensemble scolaire Saint-Étienne, qui regroupe Anne-de-Méjanès et la Miséricorde). Si elle n'a pas la prétention d'être exhaustive, cette étude s'intéresse aux lycées publics présents sur le «ban communal ${ }^{1}$ » messin, en dégageant les points les plus saillants de la création et de l'évolution de ces établissements, entre enjeux locaux et perspectives nationales, vocation d'accueil et de formation et problématiques patrimoniales.

\section{Entre France et Allemagne...}

2 Parmi les lycées publics, un seul se situe dans ce que nous pourrions appeler Metz intramuros, et il s'agit du plus ancien. De fait, le lycée impérial de Metz - actuel lycée Fabert - fut créé le 6 mai 1803 (décret du 16 floréal an XI). Logé dans l'ancienne abbaye bénédictine Saint-Vincent, sur un terrain bordant la Moselle, il ouvre ses portes en 1804. À l'ombre bienveillante des clochers gothiques de la basilique Saint-Vincent, les bâtiments conventuels sont réhabilités pour accueillir au mieux les jeunes garçons et leurs professeurs, qui s'installent également dans les bâtiments de l'ancien hôpital Saint-Georges. Après la destruction d'un pavillon, la suppression des cellules des moines et l'aménagement de salles de classe, un exhaussement est envisagé en $1854^{2}$, sans être réalisé. En 1845, la construction par l'architecte Charles Gautiez (1809-1856) d'un nouveau corps de bâtiment (le Petit Lycée, qui accueille les « petits » des classes 
élémentaires et de sixième) constitue déjà un agrandissement substantiel, prolongé quelques années plus tard avec l'acquisition d'un corps de bâtiment rue Goussaud.

Avec la signature du traité de Francfort en 1871, Metz et une partie de la Lorraine sont annexées à la Prusse. Si durant les premières années de l'Annexion la ville doit faire face à l'exode d'une partie de sa population, une nouvelle dynamique s'installe dans bien des domaines à mesure qu'approche l'aube $\mathrm{du} \mathrm{xx}^{\mathrm{e}}$ siècle. Que ce soit en termes d'activité économique, culturelle, de construction ou encore d'urbanisme, Metz se replace dans un mouvement positif qui avait été stoppé brutalement par les bouleversements entraînés par l'Annexion et l'exil de la quasi-totalité des élites locales.

Avec la reprise de l'accroissement de la population locale, scolariser les plus jeunes nécessite la construction de quelques écoles, dessinées par les architectes allemands dans un style pseudo-local, réminiscence du Moyen Âge ou encore de la Renaissance, époques durant lesquelles Metz avait des liens avec l'Empire germanique. Le courant Heimatschutz, qui défend la préservation des particularismes architecturaux régionaux, gagne l'architecture des écoles primaires.

5 Le lycée, devenu le Kaiserliches Lyceum ${ }^{3}$ [fig. 1], continue pour sa part d'accueillir de jeunes garçons. Des travaux importants y sont engagés, comme la construction d'une salle des fêtes et d'une salle de gymnastique (1883), en lieu et place de la partie ouest du cloître, démolie pour accueillir ce nouveau programme ${ }^{4}$.

Figure 1

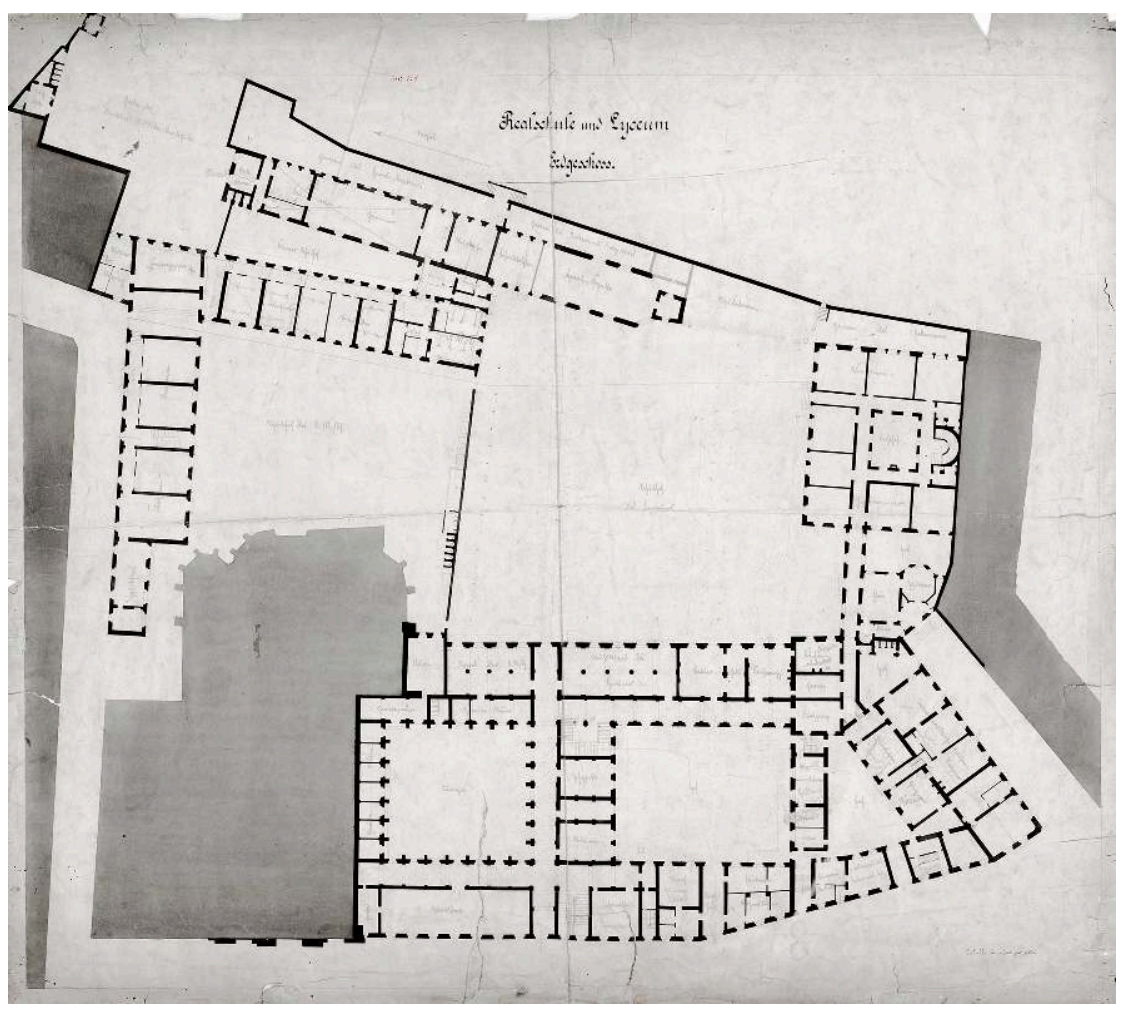

Plan du rez-de-chaussée de la Realschule et du Lyceum (actuel lycée Fabert) durant l'Annexion allemande, non daté, conservé aux archives municipales de Metz (9Fi691).

Reproduction archives municipales de Metz.

6 Si aucun Gymnasium n'est construit ou aménagé à Metz durant l'Annexion, on peut toutefois noter l'édification ex nihilo de deux établissements dans l'extension sud de 
la ville ${ }^{5}$. Le premier est l'école supérieure de jeunes filles (Höhere Mädchenschule ${ }^{6}$, actuel lycée Georges-de-La-Tour), destinée à remplacer un établissement trop exigu du centre-ville de Metz. Pour concevoir le meilleur projet, l'architecte Conrad Wahn (1851-1927), un professeur et le directeur de l'école effectuent un voyage en Allemagne en octobre 1905, au cours duquel ils visitent des écoles récemment construites à Strasbourg, Mannheim, Darmstadt, Wiesbaden ou encore Essen ${ }^{8}$. Éclairé par ces références et sans doute aussi par le plaidoyer de médecins, pédagogues et architectes allemands du début du $\mathrm{xx}^{\mathrm{e}}$ siècle en faveur d'une architecture hygiénique, lumineuse, colorée et bien ventilée ${ }^{9}$, Conrad Wahn esquisse un projet architectural qui est présenté au conseil municipal en janvier 1906 [fig. 2].

Figure 2

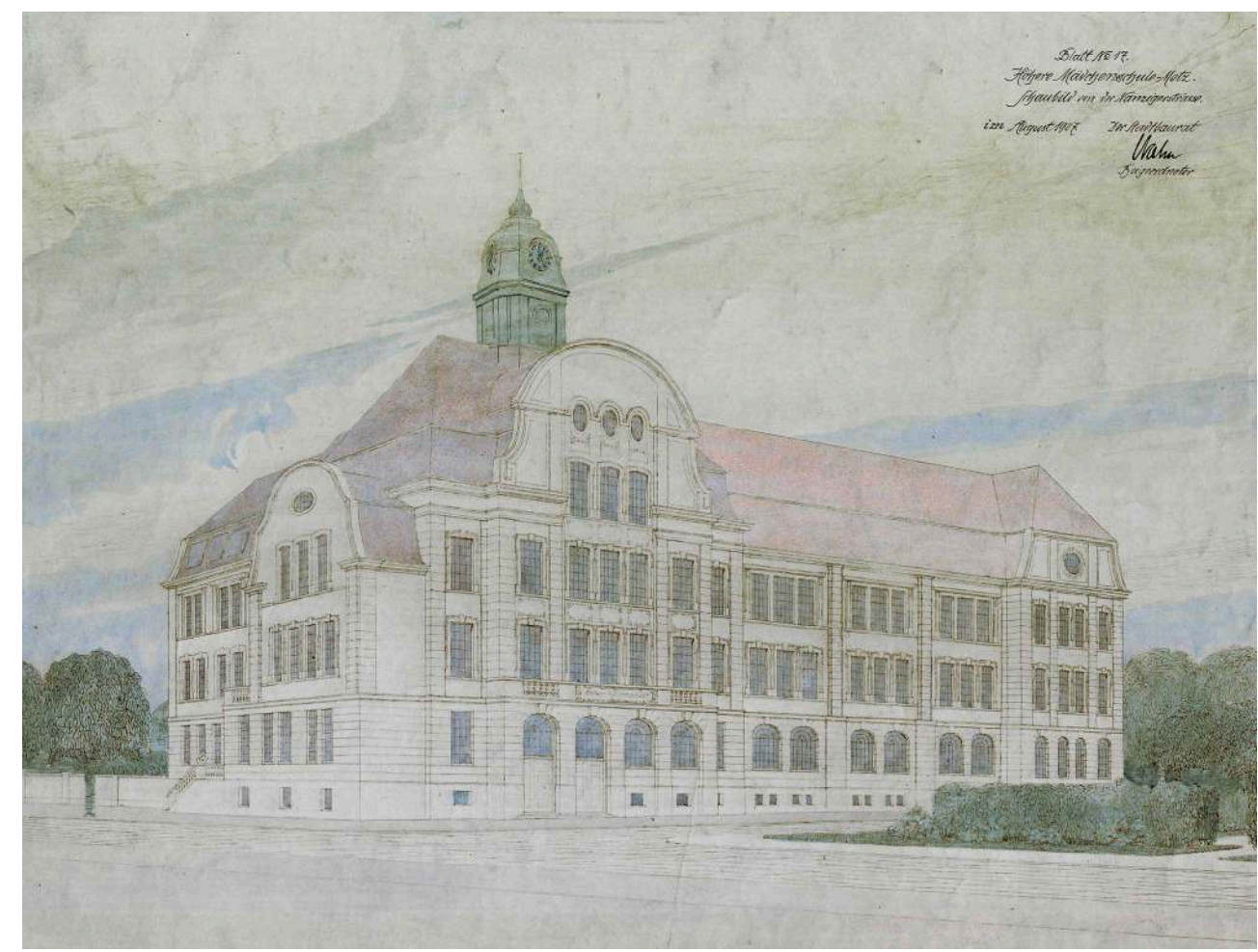

Étude pour la Höhere Mädchenschule (futur lycée de jeunes filles, actuel lycée Georges-de-La-Tour) par Conrad Wahn, 1907, conservé aux archives municipales de Metz (9Fi1729).

Reproduction archives municipales de Metz.

7 Les travaux commencent la même année avec la construction de la maison du directeur, achevée l'année suivante. Le corps de bâtiment principal est ensuite édifié de juin 1908 à mars 1910 et l'école est inaugurée durant l'été 1910. Massive, cette école présente une façade principale de près de 70 mètres de large pour 65 mètres pour les deux ailes latérales [fig. 3] ; les salles de classe, donnant sur l'extérieur, sont desservies par un couloir de 7 mètres de large. 


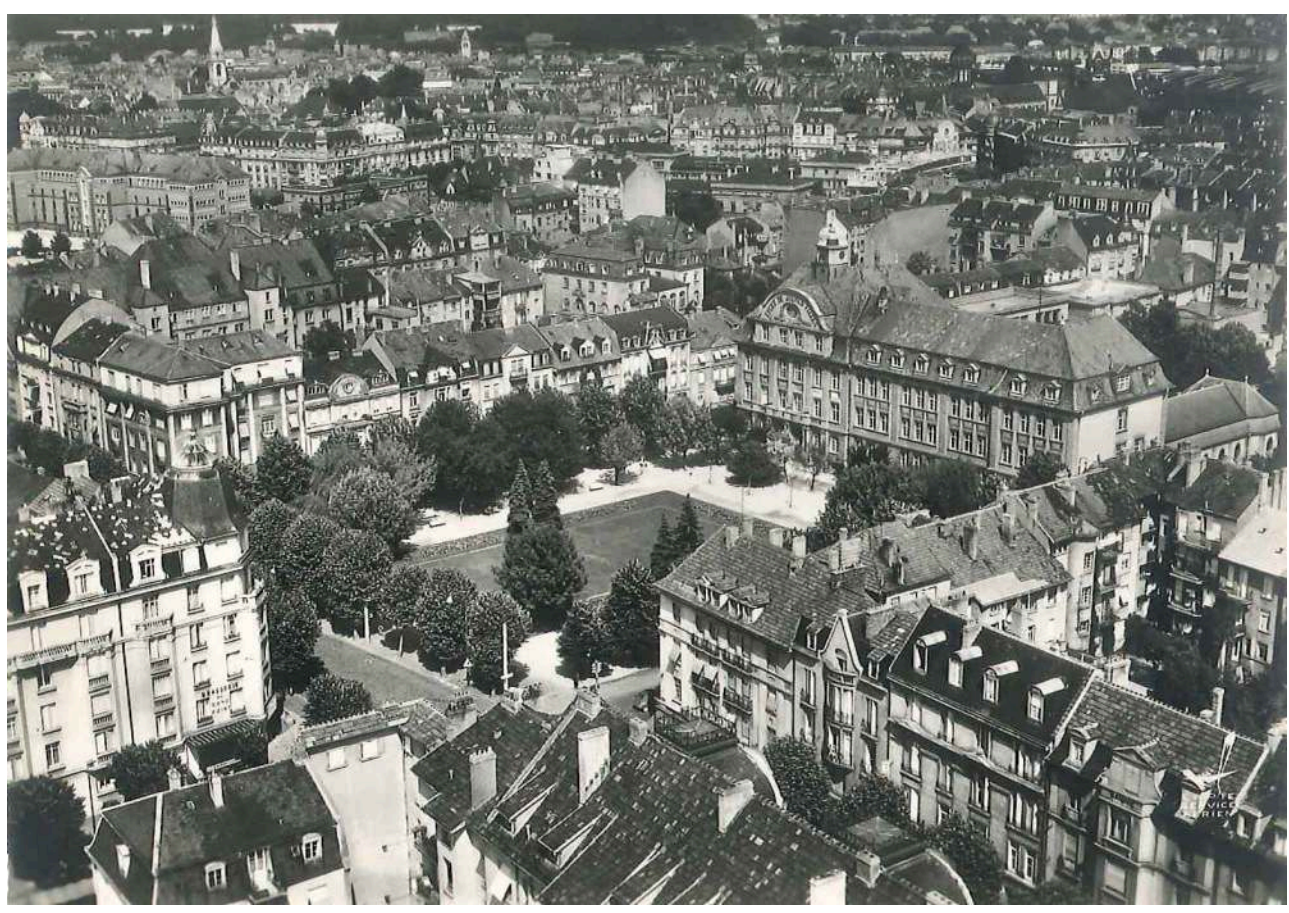

L'ancienne Höhere Mädchenschule (actuel lycée Georges-de-La-Tour) vue d'avion dans les années 1950, carte postale. Dans le coin supérieur gauche, on aperçoit une partie de la cité scolaire Barbot, implantée dans les années 1950 dans d'anciennes casernes. Collection privée.

Reproduction Pierre Maurer.

Se développant sur trois niveaux, les façades en moellons et pierre de taille reprennent une écriture très classique, bien qu'elles soient décrites comme « des formes baroques modernes et simples » dont le « caractère doux et serein » est censé « convenir à une école de jeunes filles ${ }^{10} »$. Si le souhait de ses concepteurs est de rappeler l'" architecture lorraine locale ${ }^{11} »$, l'édifice semble également inspiré par des constructions de Sarre ou du Palatinat ou encore de Trèves et de Coblence ${ }^{12}$. Les dimensions imposantes de cette école sont accentuées par le couronnement constitué par une vaste couverture mansardée. La façade refuse la symétrie et l'entrée est placée parmi les travées gauches, marquées par un avant-corps dont les pilastres cannelés sont surmontés par un fronton courbe derrière lequel se dessine un lanternon de cuivre. Le décor baroque de l'entrée de la salle des fêtes et le décor Jugendstil de l'entrée principale contribuent à faire de cette construction une œuvre singulière. Notons enfin que la distribution intérieure est conforme aux normes spatiales et hygiénistes édictées en Allemagne ${ }^{13}$.

9 Après la construction de cet établissement pour jeunes filles, Conrad Wahn et les services municipaux œuvrent à une nouvelle réalisation scolaire destinée aux garçons, et d'enseignement, cette fois, professionnel. Ici encore, la construction est massive, les références convoquées étant les mêmes que pour le précédent établissement, avec la volonté de présenter « un baroque moderne faisant référence aux modèles locaux ${ }^{14}$ ». Les travaux de l'école supérieure de garçons (Oberrealschule, actuel lycée Louis-Vincent) démarrent en 1912 et sont achevés en plein conflit mondial, en 1916.

10 En matière stylistique, les lycées construits pendant l'Annexion se révèlent très différents des écoles primaires et de leur style pseudo-local. On notera la permanence de l'utilisation de la pierre calcaire de Jaumont, emblématique de Metz, et le souci 
d'intégrer ces réalisations massives dans le paysage messin, à l'image de la nouvelle façade du Kaiserliches Lyceum sur la place Saint-Vincent, incluse dans le cadre de la construction de la salle des fêtes [fig. 4].

Figure 4

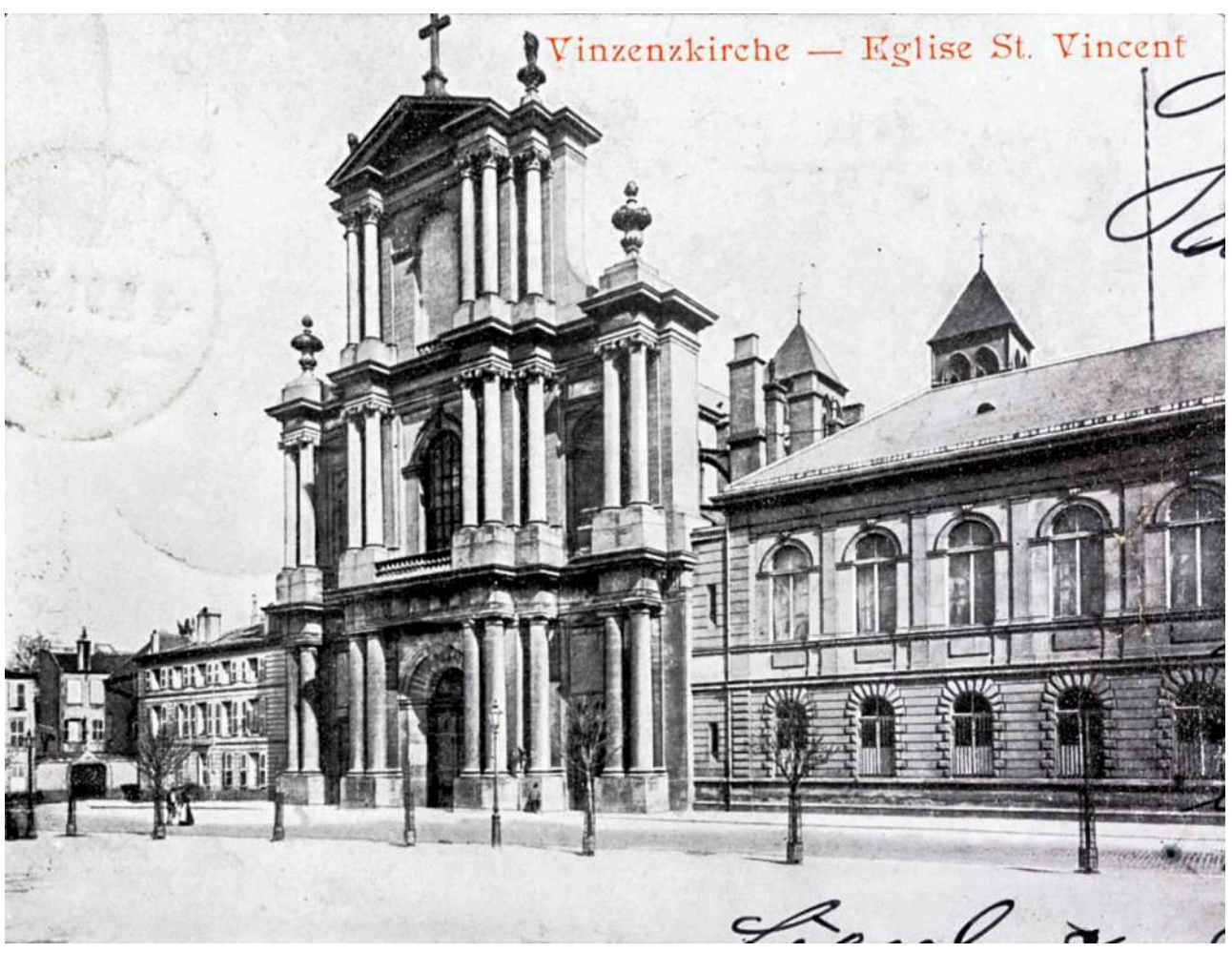

La basilique Saint-Vincent et l'extension allemande du Kaiserliches Lyceum abritant la salle des fêtes (sur la droite), carte postale, vers 1900, éditée par Stern \& Löb (Francfort), conservée aux archives municipales de Metz (4Fi397).

Reproduction archives municipales de Metz.

\section{L'entre-deux-guerres ou le temps des extensions}

Une fois l'armistice signé et Metz redevenue française, la ville doit s'adapter au système d'enseignement français. Le Kaiserliches Lyceum devient lycée de garçons et la Höhere Mädchenschule lycée de jeunes filles.

Si les années dites de l'entre-deux-guerres ne sont pas marquées par la construction d'un troisième établissement, les deux lycées existants font durant cette période l'objet de travaux d'extension pour répondre au mieux aux besoins de ces établissements qui trouvent vite leurs limites spatiales dans leur configuration d'origine ${ }^{15}$. En parallèle, la nationalisation des lycées d'Alsace-Lorraine, jusqu'alors gérés par les communes, est engagée et devient effective en 1930.

Divers aménagements sont entrepris au lycée de garçons, à commencer par le décloisonnement des arcades du cloître en $1932^{16}$, la pose de nouvelles portes par le ferronnier Edgar Brandt (1880-1960) ou encore la création d'une œuvre d'art ${ }^{17}$ par le peintre Nicolas Untersteller ${ }^{18}$ (1900-1967), dans le parloir. Mais c'est bien le manque de place et la nécessité de faire face à l'accroissement des effectifs qui poussent la Ville et l'établissement - porté par la détermination du proviseur Émile Chamoux, récemment 
nommé à Metz pour mettre en œuvre la nationalisation du lycée - à s'engager dans la construction d'une annexe. La commune acquiert ainsi une ancienne caserne, située à proximité dulycée, dont elle obtient la démolition. La dénommée «caserne du Cloître ", très délabrée, comporte alors une porte et une fenêtre datant $\mathrm{du}$ XvII ${ }^{\mathrm{e}}$ siècle, vestiges du bâtiment originel, un couvent d'Ursulines. Ces deux éléments remarquables viennent tout juste d'être classés au titre des monuments historiques, le 30 mars 1926, à l'initiative de l'architecte du gouvernement, Ernest Herpe, qui s'inquiétait de leur éventuelle disparition alors que l'administration domaniale mettait en vente la caserne. Si cette protection suscite différents échanges et même une demande de déclassement $\mathrm{du}$ maire, la commission des Monuments historiques s'était engagée, lors du classement, à ne pas s'opposer à un projet de démolition à condition que les deux éléments classés soient conservés. Ainsi la caserne disparait-elle et les deux éléments protégés sont censés être incorporés au nouveau projet ${ }^{19}$. De fait, le site est alors destiné à accueillir l'internat du lycée et un concours architectural est ouvert. Le 4 juin 1932, le projet porté par Robert Parisot (1897-1965) et Paul Millochau (1904-vers 1973) est déclaré lauréat [fig. 5].

Figure 5

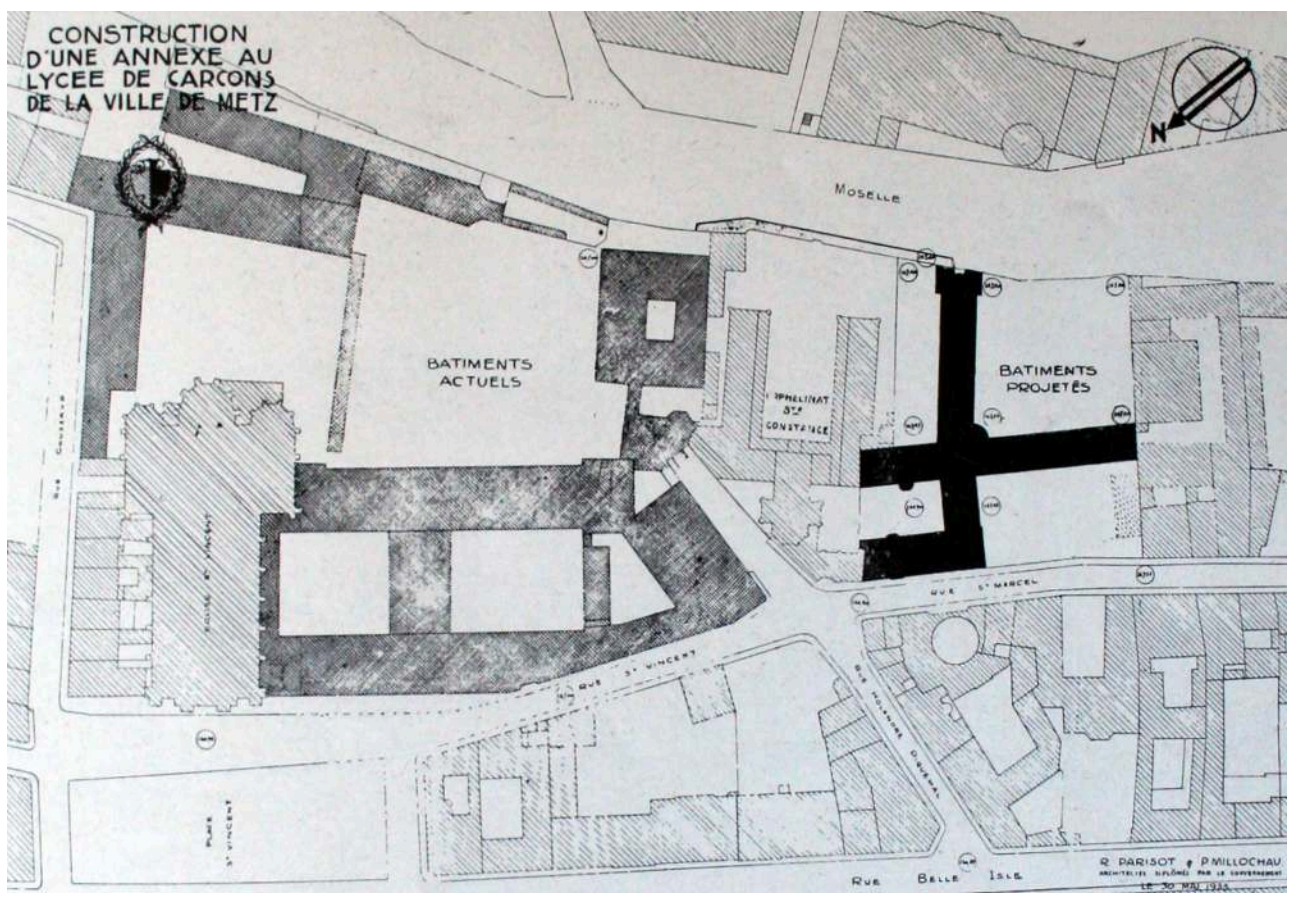

Plan-masse du lycée de garçons (actuel lycée Fabert) et de son internat par Robert Parisot et Paul Millochau, 30 mai 1933. Extrait de l'article d'Albert Laprade, «L'Architecture dans nos provinces françaises. Le Lycée de Metz, R. Parisot et P. Millochau, architectes », L'Architecture, n 7, juillet 1937, p. 229.

Reproduction Pierre Maurer.

Faisant confiance à des architectes non messins (un Nancéien et un Parisien), jeunes diplômés, le jury du concours fait preuve d'une certaine audace, qui se ressent dans le projet lui-même. Si ce dernier est remanié pour être finalement validé en 1934, ses lignes architecturales sont alors très nouvelles à Metz : l'édifice est très élancé, et ses façades en brique finement dessinées présentent des motifs en croisillon [fig. 6]. 


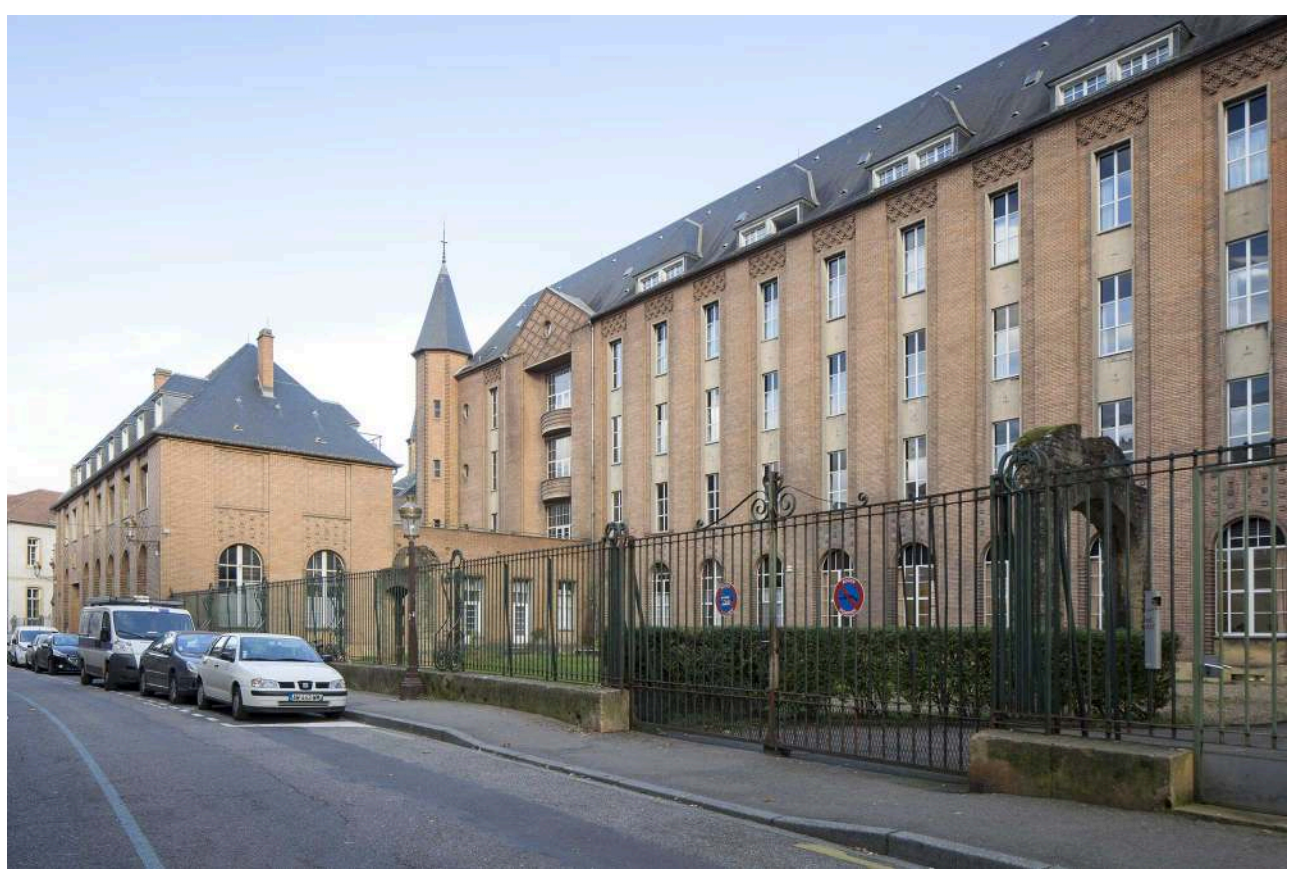

L'internat du lycée de garçons (actuel lycée Fabert) : dans la cour, les deux baies protégées au titre des Monuments historiques.

(c) Olivier Mathiotte (La Manufacture du patrimoine)

Certains enseignants reprochent d'ailleurs au proviseur Chamoux ce choix trop peu local - la pierre de Jaumont, qui habille l'ensemble de la ville de Metz, aurait sans nul doute fait davantage consensus. Comme l'expliquera plus tard l'un des élèves du lycée, «à juste titre, on reprocha aux nouveaux bâtiments, édifiés en brique et coiffés de toits d'ardoise fortement pentus, d'être aussi peu messins que possible ${ }^{20}$ ». Pourtant, l'édifice, si différent soit-il de ses voisins, s'inscrit avec une certaine finesse dans son site, dégageant des cours et respectant les gabarits des immeubles voisins, une mince tour étant même insérée comme en écho aux tourelles voisines del'hôtel du Passetemps et de la chapelle Sainte-Constance. Ne pouvant être incorporées dans les murs de brique, les deux baies classées de la caserne du Cloître sont remontées devant l'internat, dans une cour donnant directement sur la rue Saint-Marcel, ce qui leur offre un cadre certes qualitatif mais leur fait perdre toute valeur d'usage en les transformant en objets d'architecture, à mi-chemin entre ruines et sculptures.

L'internat ouvre le $1^{\mathrm{er}}$ octobre 1936 et les boxes accueillant les internes sont dotés d'un mobilier conçu spécialement par les ateliers du créateur Jean Prouvé (1901-1984).

La fortune critique de cette nouvelle construction est assez immédiate. La diffusion d'un album intitulé L'Internat du lycée de Metz ${ }^{21}$, présentant son architecture, concourt à en faire une des références françaises pour ce type de programme. La presse spécialisée s'en fait largement l'écho, de L'Architecture d'aujourd'hui ${ }^{22}$ à L'Architecture ${ }^{23}$, en passant par La Construction moderne ${ }^{24}$ ou encore Art national construction ${ }^{25}$. Robert Parisot et Paul Millochau remportent en 1936 le premier prix de l'académie d'Art national, décerné par la revue Art national construction, pour l'internat du lycée de garçons ${ }^{26}$.

Comme le lycée de garçons, le lycée de jeunes filles accueille non seulement l'enseignement secondaire mais également les classes maternelles et primaires. Ses locaux s'avèrent bien vite insuffisants et la direction de l'établissement demande à la 
commune que soient réalisés d'une part des travaux d'extension de l'externat et d'autre part la construction d'un internat. Si les différentes propositions pour la construction de l'internat n'aboutissent pas, une extension du lycée sur la rue Rabelais est néanmoins réalisée. Comme pour l'internat du lycée de garçons, c'est une construction en briques sans doute inspirée par d'autres réalisations françaises contemporaines ${ }^{27}$, sans que les architectes ne donnent cependant de raison précise sur ce choix de matériau. Théophile Dedun (1878-1967), architecte local ayant la particularité d'avoir œuvré à Metz durant l'Annexion et d'avoir poursuivi son activité après le retour à la France (alors que la quasi-totalité des architectes, allemands, fut contrainte de quitter Metz pour rentrer en Allemagne), conçoit un projet en 1932. Le chantier est rapidement engagé et dure jusqu'en 1933. Cette construction sobre et élégante est composée de deux corps de bâtiment dans le prolongement l'un de l'autre, reliés au niveau du rezde-chaussée, qui s'insèrent pour l'un au niveau du faîtage du lycée voisin et pour l'autre à celui de l'immeuble mitoyen. Un jeu entre des briques jaunes, orangées et rouges rend cette façade dynamique et contrebalance la rigueur de sa composition [fig. 7].

Figure 7

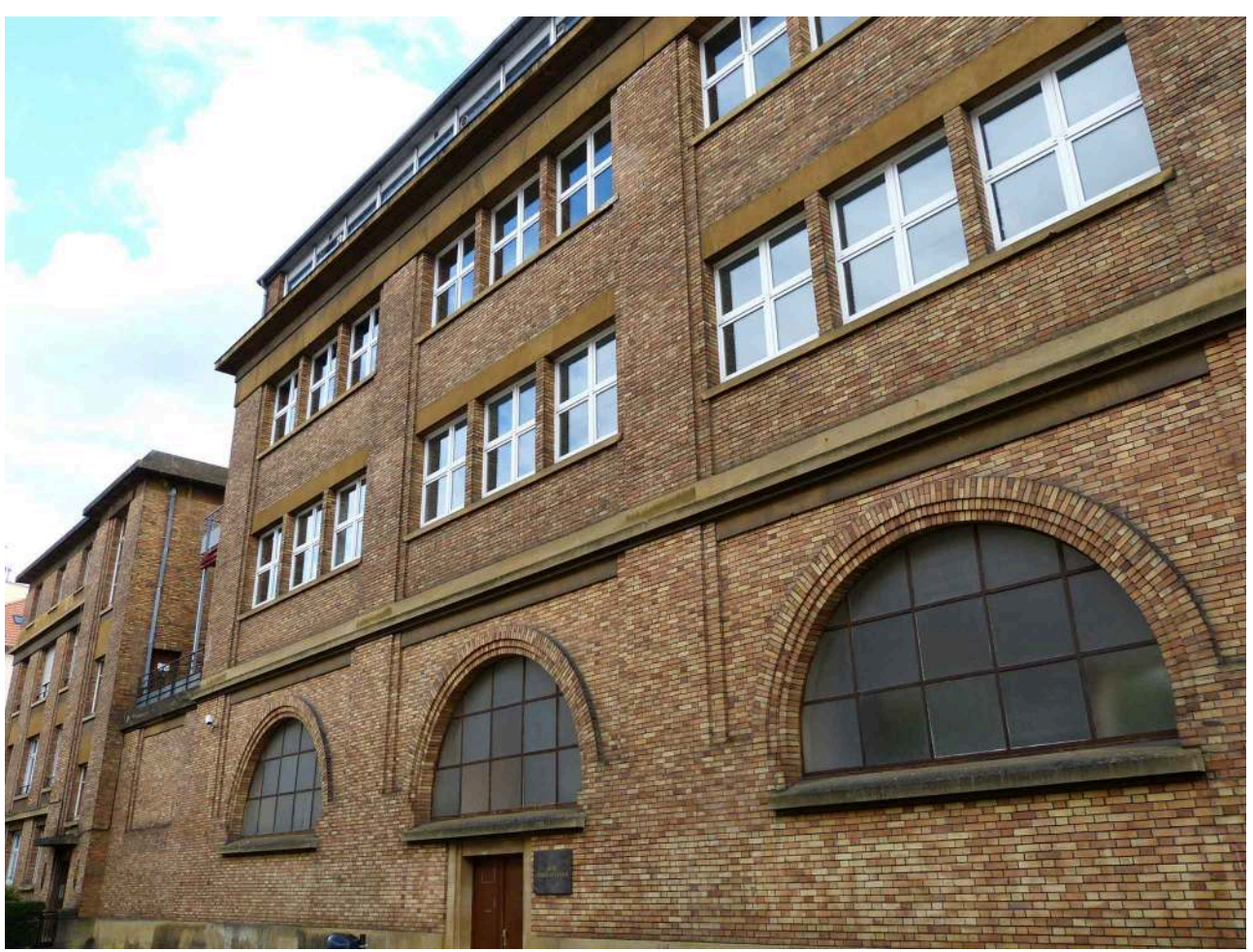

L'extension du lycée de jeunes filles (actuel lycée Georges-de-La-Tour) par Théophile Dedun, 1933.

(c) Christiane Massel.

Par sa relative modestie et le raffinement simple de ses façades, cette extension est une réponse contemporaine au bâtiment néo-baroque du lycée ${ }^{28}$. En un sens, elle cristallise la volonté des architectes de l'entre-deux-guerres de se distinguer de l'époque précédente par l'emploi d'autres matériaux et de formes non historicistes. Signalons également les extensions de l'ancienne Oberrealschule, devenue École nationale professionnelle, et tout spécialement la construction de l'internat (1934-1938) par Robert Fournez (1873-1958) et Louis Sainsaulieu (1901-1972), construction en béton 
armé qui se détache intelligemment du bâtiment principal et assume une écriture architecturale bien plus sobre mais non moins forte ${ }^{29}$.

\section{Des conséquences du baby-boom et de la démocratisation de l'enseignement secondaire en France}

Après la Seconde Guerre mondiale, le baby-boom a une incidence directe sur la construction d'établissements scolaires et en particulier sur les écoles maternelles et primaires, suscitant la création de dizaines d'édifices sur le ban communal messin. Comme sur le plan national, ces constructions se caractérisent par une rationalisation de l'architecture et une industrialisation qui ont un impact direct sur l'aspect formel de ces édifices, largement réalisés avec des éléments préfabriqués. De 2465 élèves en 1948, les lycées de garçons et de filles voient passer leurs effectifs à 3694 en $1958^{30}$. De plus, en janvier 1959, la réforme Berthoin ${ }^{31}$ rend la scolarisation obligatoire jusqu'à seize ans révolus. Cette croissance des effectifs rend donc nécessaire une réévaluation des capacités d'accueil de la population lycéenne sur le ban communal.

En 1946, des baraques-dortoirs sont édifiées à quelques centaines de mètres du lycée de jeunes filles - qui prend par la suite pour nom "lycée Georges-de-La-Tour»-pour accueillir les internes ${ }^{32}$, avant qu'un internat plus pérenne soit aménagé dans la nouvelle cité scolaire Barbot.

Robert Parisot et Paul Millochau, qui auraient dû construire le nouvel externat du lycée de garçons avant le début du conflit mondial, sont chargés de concevoir un nouveau projet afin de remplacer les principaux bâtiments endommagés pendant la bataille de novembre $1944^{33}$. Entamés en $1954^{34}$, les travaux de construction de la première aile du nouveau bâtiment de l'externat sont achevés en $1956^{35}$. Une deuxième aile est construite entre 1959 et 1960, avant que de nouveaux travaux soient réalisés entre 1962 et $1963^{36}$. De plus, l'orphelinat Sainte-Constance, situé entre le lycée et l'internat, est acheté et intégré au lycée, qui prend par ailleurs en 1962 son nom actuel "lycée Fabert $^{37}$ ». Si le lycée de garçons / Fabert regroupe plusieurs établissements sous sa tutelle dans la ville de Metz ou même le département de la Moselle, ces derniers sont amenés à (re)prendre leur autonomie, bien souvent en accord avec l'évolution statutaire de ces lieux d'enseignement. Ainsi, le premier cycle du lycée devient par décret du 20 décembre 1974 le collège d'enseignement secondaire (CES) de MetzArsenal ${ }^{38}$. En ce qui concerne la mixité, il faut attendre la rentrée 1971 pour que des filles soient scolarisées en classe de sixième et de seconde ${ }^{39}$.

Alors que le lycée de garçons a une capacité d'accueil de 1000 à 1200 élèves, plus de 2000 y sont inscrits dès 1955 . Le projet de création d'un nouveau lycée pouvant accueillir 1500 élèves est donc soumis et accepté par l'État, qui laisse néanmoins le soin à la commune de trouver le terrain adéquat ${ }^{40}$. Elle trouve ce dernier sur les hauteurs sud-est de Metz. En plus de l'apport du terrain, la Ville doit également financer la construction de l'externat, avec subvention de l'État, ce dernier prenant entièrement en charge le coût de l'internat ${ }^{41}$. Ce montage financier est toutefois remis en question dans les années qui suivent, l'État souhaitant que la commune contribue davantage financièrement, compte tenu de l'évolution de la réglementation. S'appuyant sur les 
engagements initiaux, la Ville parvient à se soustraire à une partie des exigences de l'État ${ }^{42}$.

Si la commune choisit les architectes Max Doignon-Tournier (1922-1986) et Louis Pitet (1915-1989) pour concevoir ce nouveau lycée, l'État impose pour sa part la supervision de Bertrand Monnet (1910-1989), chargé d'en coordonner la construction.

Le projet est arrêté dans ses grandes lignes au début des années 1960 et la construction est alors prévue en trois phases menées presque simultanément ${ }^{43}$. Parmi les ajustements dont doit s'accommoder le projet initial figure l'implantation sur le même site d'un lycée canadien - les forces armées de l'Otan étant alors stationnées en Lorraine - dont l'étude est engagée en avril 1962; inauguré pour la rentrée 1963, il ferme en 1967, au départ des troupes canadiennes ${ }^{44}$.

Bien que retardé par la longueur et la rigueur de l'hiver 1962-1963 ${ }^{45}$, le chantier de construction progresse et le lycée peut ouvrir ses portes en septembre 1963. Inauguré en avril 1964 par le ministre de l'Éducation nationale Christian Fouchet $^{46}$, il prend le nom de Robert Schuman (1886-1963), l'un des initiateurs de la construction politique européenne, décédé quelques mois auparavant.

Si l'ouverture de ce nouveau lycée classique et technique entraîne une réorganisation de l'ensemble des établissements d'enseignement secondaire messins, la mixité qu'il généralise marque également une grande nouveauté dans l'organisation scolaire ${ }^{47}$.

Dans un vaste site d'une trentaine d'hectares, différents corps de bâtiment constituent l'internat et l'externat du lycée [fig. 8], auxquels s'ajoutent d'autres lieux d'enseignement secondaire et supérieur, des espaces et salles de sport et une piscine.

Figure 8

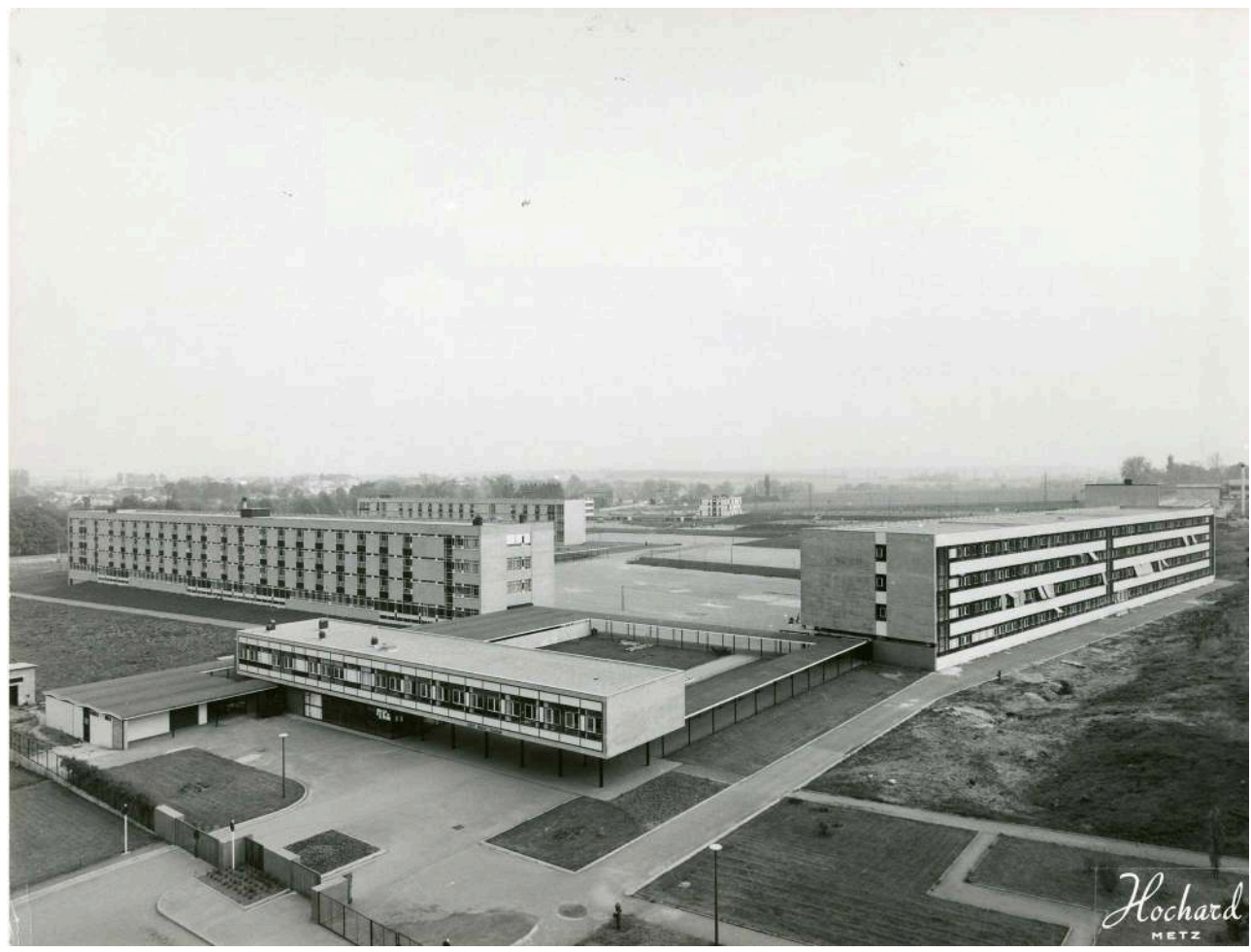

Le lycée Robert-Schuman, carte postale éditée par Hochard (Metz), années 1960, conservée aux archives municipales de Metz (1Fi137).

Reproduction archives municipales de Metz. 
L'ensemble est construit avec des éléments d'architecture préfabriqués, la rationalisation de la construction et la répétition des façades et des trames trouvant ici un aboutissement certain en ce qui concerne les réalisations messines. Seul le dessin des baies ou la présence d'un escalier vient perturber l'horizontalité et la linéarité des larges façades. Cet ensemble soigné aux salles de cours lumineuses [fig.9], peut accueillir plusieurs milliers d'étudiants, ce qui en fait une véritable cité scolaire.

Figure 9

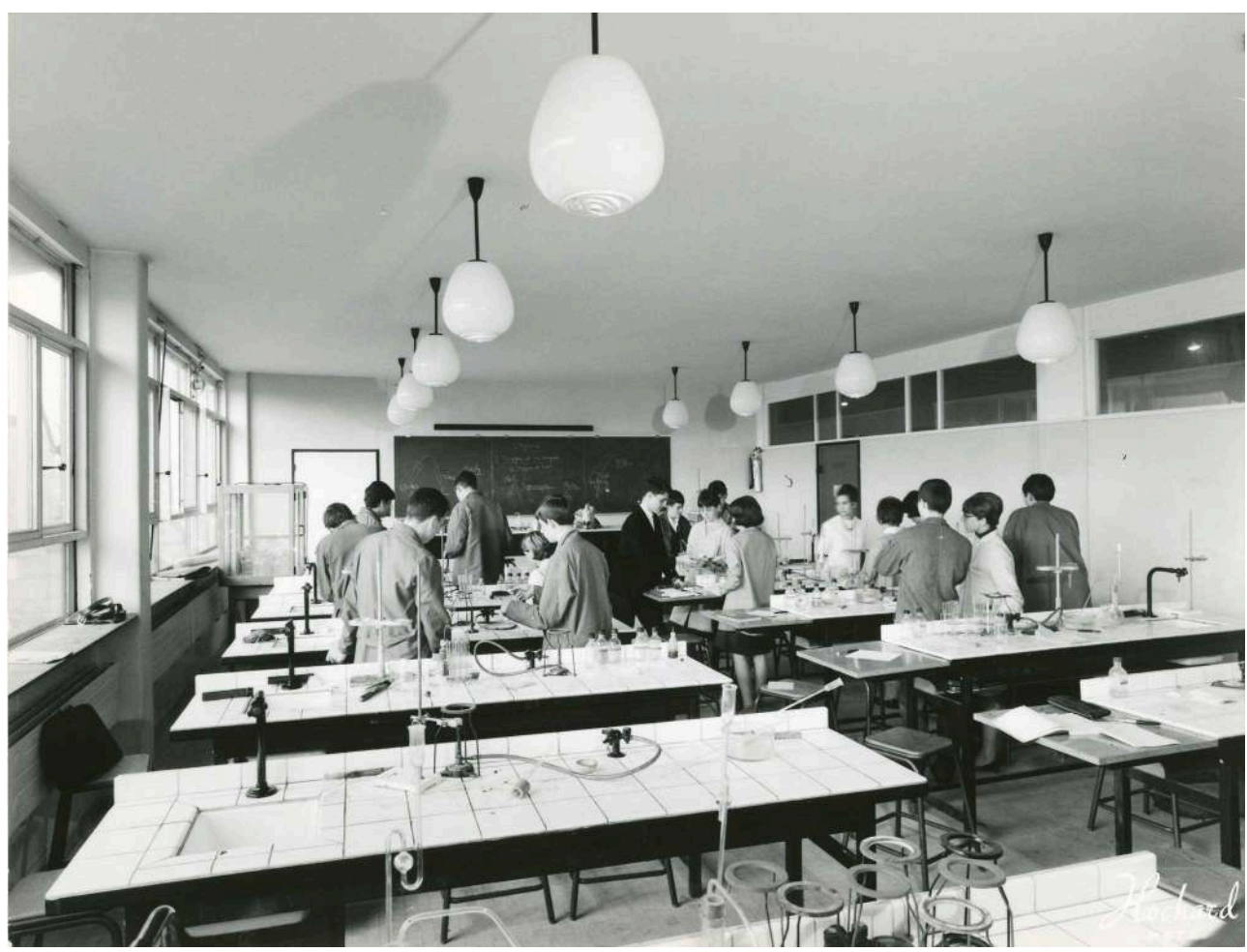

Salle de chimie du lycée Robert-Schuman, carte postale éditée par Hochard (Metz), années 1960, conservée aux archives municipales de Metz (1Fi139).

Reproduction archives municipales de Metz.

Par la suite, d'autres lycées sont encore édifiés sur le ban messin (tels le lycée hôtelier Raymond-Mondon et le lycée Jean-Victor-Poncelet). Dans le même temps, l'École nationale professionnelle devient «lycée technique Louis-Vincent » et l'ancien collège d'enseignement technique industriel et masculin de Metz devient "lycée Louis-deCormontaigne ». Quant à l'ancien centre d'apprentissage de jeunes filles (ou collège d'enseignement technique [CET] féminin Alain-Fournier), cette réalisation compacte percée de deux patios dont le toit-terrasse abrite plusieurs petits logements, il est depuis devenu le lycée technique Alain-Fournier et compte sans doute parmi les réalisations les plus intéressantes des Trente Glorieuses à $\mathrm{Metz}^{48}$.

\section{Les blanches architectures des années 1980-1990}

Durant les deux dernières décennies $\mathrm{du} \mathrm{xx}^{\mathrm{e}}$ siècle, la construction de lycées se poursuit avec, en particulier, deux projets ex nihilo. 

ensemble d'architectes réunis au sein de trois structures messines: AAG Sarl (Victor Klein directeur général, Jean Cuny, Jean-Claude Cadoux et Henri Schaack architectes), Alpha Architecture (Jean-Luc Gibert et Gérard Hypolite architectes) et SCPA (Michel Klein et Jean Denis Sagan architectes). Dans le cadre du projet de décentralisation engagé en 1982, l'État transfère la compétence de la construction et de la gestion des lycées aux régions à compter du $1^{\mathrm{er}}$ janvier $1986^{49}$. Maître d'ouvrage, la région Lorraine - présidée entre 1982 et 1992 par Jean-Marie Rausch, maire de Metz et ministre dans les gouvernements de Michel Rocard et Édith Cresson - confie la réalisation de ce lycée à la Ville de Metz, sous la forme d'une convention de mandat.

Le permis de construire est déposé en mars 1988 et les travaux sont engagés dès l'été 1988, la réception des bâtiments étant organisée un an plus tard, pour que les nouveaux lycéens puissent y faire leur première rentrée dès le 4 septembre 1989 [fig. 10].

Figure 10

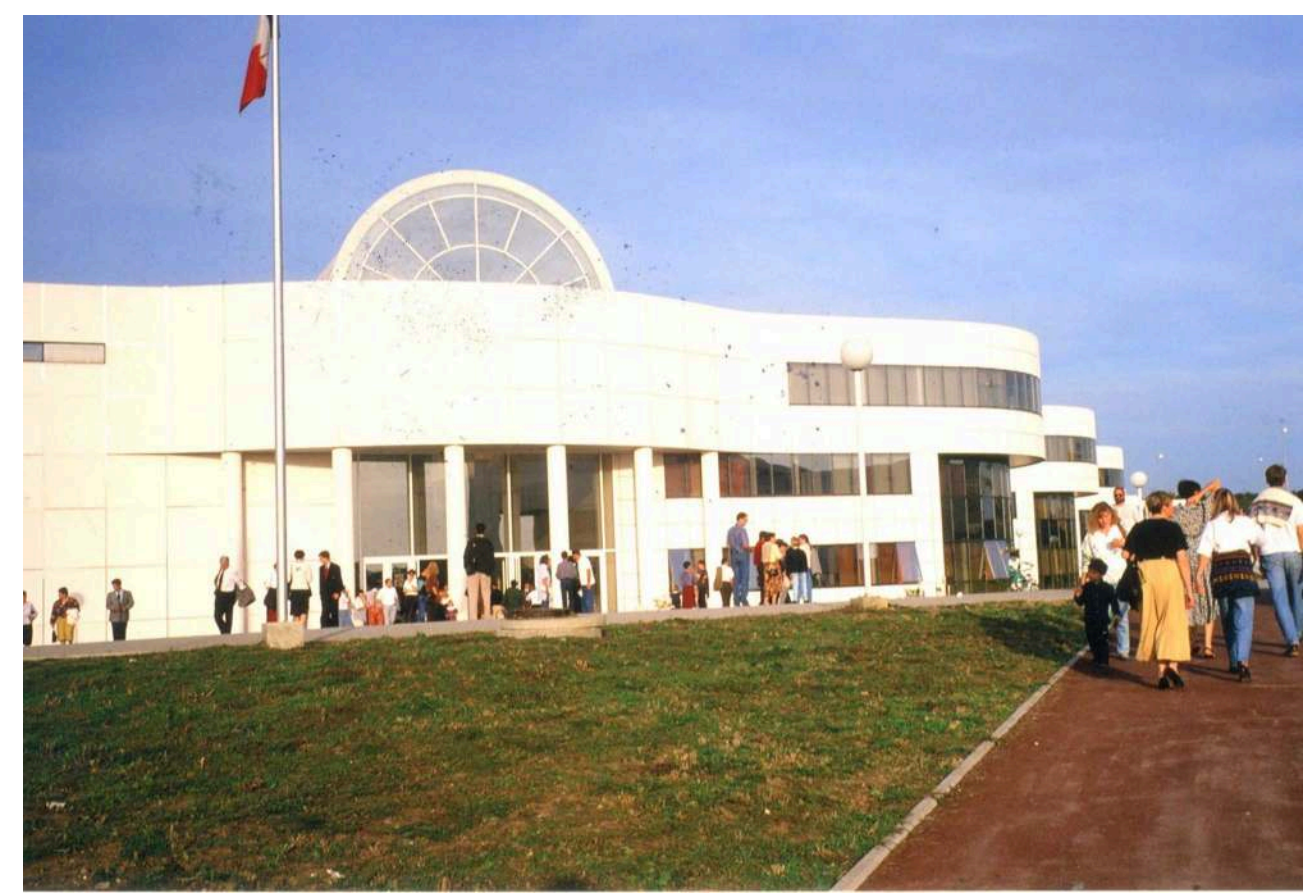

Le lycée de la Communication, entrée principale photographiée vers 1990, conservée aux archives municipales de Metz (2Fi1608).

Reproduction archives municipales de Metz.

Il faut néanmoins attendre fin 1988 pour que l'établissement soit définitivement réceptionné. D'une capacité d'accueil de près de 850 places, le lycée doit permettre de faire face à la croissance des effectifs lycéens prévue à l'horizon $1992^{50}$. S'inscrivant au cœur du Technopôle Metz 2000, il répond à l'ambition fixée à ce parc d'activités qui est de "faire de la Lorraine un pôle d'excellence en matière de communication » en regroupant «sur un même site des entreprises, des unités de recherche et des organismes de formation avec comme dénominateur commun le domaine de la communication $^{51} »$. 

à un projet sur le thème de la communication dont le symbole le plus évident est la main: "Main tendue, poignée de main, autant d'images qui sont les premiers maillons de la chaîne communication. La main, en plus de la symbolique, est aussi un fonctionnement ${ }^{52}$. » Le concept se traduit au plan spatial par un plan-masse composé de trois corps de bâtiment longilignes parallèles surnommés "les doigts » qui abritent l'enseignement général et sont reliés par des espaces de circulation. Le pouce est un triangle accueillant départements et ateliers, la paume, cœur structurant du projet, est une grande rue intérieure [Fig.11] animée par des passerelles, des coursives et la présence de la médiathèque. Un internat de 144 places vient se greffer à cette main.

\section{Figure 11}

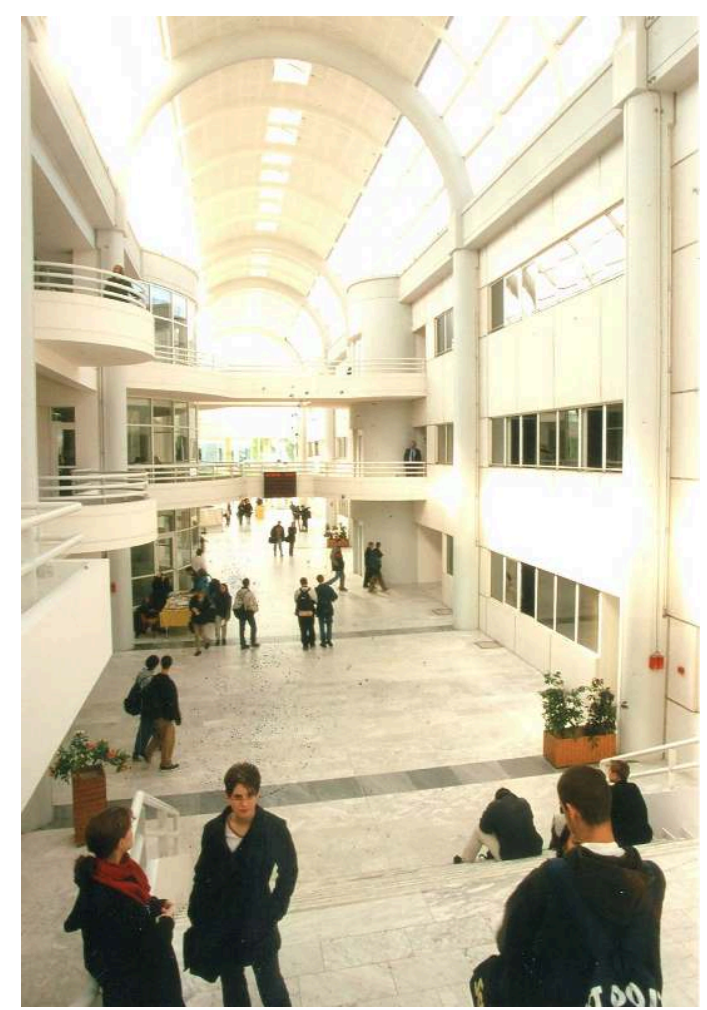

La grande rue intérieure du lycée de la Communication, photographiée vers 1990, conservée aux archives municipales de Metz (2Fi1608).

Reproduction archives municipales de Metz.

Peu de temps après l'ouverture du lycée de la Communication, la Région Lorraine envisage la création d'un nouvel établissement. Pour désengorger le lycée RobertSchuman et en particulier, son offre de formation professionnelle, un projet de construction de lycée au nord de la commune émerge au début des années $1990^{53}$. Une nouvelle fois, la Ville de Metz est mandatée pour assurer la maitrise d'ouvrage pour son compte et mettre en œuvre ce projet de « lycée professionnel régional du commerce et de la distribution " prévu pour accueillir 1050 élèves sur le site d'un ancien dépôt des transports en commun, dans les faubourgs nord de la ville.

Une consultation architecturale est lancée en novembre 1991 avec la publication dans la presse d'un appel à candidature. Trois équipes d'architectes locaux sont sélectionnées en décembre, qui transmettent chacune une offre en mars 1992, le choix 
du titulaire se faisant le 12 du même mois. La proposition retenue est celle de Michel Klein et Jean Denis Sagan, architectes ayant déjà fait partie de l'équipe de maitrise d'œuvre dulycée de la Communication. Le permis de construire est déposé en juin 1992, avec la volonté d'achever les travaux début août 1993, après onze mois de travaux ${ }^{54}$. Néanmoins, le chantier ne commence qu'au printemps $1993^{55}$ et le nouveau lycée, dénommé « lycée professionnel régional René-Cassin », ouvre à la rentrée 1994.

Ces deux lycées ont en commun d'avoir été conçus par des architectes locaux, qui proposent ici des architectures essentiellement blanches, aux formes simples répondant à une force conceptuelle ancrée dans un discours qui offre une large place au cheminement architectural. La structure du lycée René-Cassin allie le bois en lamellé-collé avec un système de poteaux-poutres en béton armé qui forme l'ossature du bâtiment. Le parement des façades en béton à base de résine donne à l'ensemble une unité et crée une cohésion entre les différents corps de bâtiment. De fait, deux ensembles se font face et sont reliés entre eux par un système de passerelles. Le concept original des architectes repose en effet sur la mise en place d'un cheminement central entre ces deux ensembles, qui devaient être publics à l'origine, de sorte à « faire passer la vie quotidienne de façon [...] symbolique au milieu du lycée [dans une] volonté de faire cohabiter ces deux milieux souvent fermés l'un à l'autre ${ }^{56}$ ». La volonté des architectes est ainsi d'humaniser les lieux d'enseignement. Autour de cet axe central structurant, ils prennent la liberté de jouer "sur la qualité d'une architecture rigide en opposition à une architecture aux formes plus souples ${ }^{57}$ ».

\section{Un patrimoine vivant}

Anne-Marie Châtelet a démontré combien l'architecture scolaire était un patrimoine inégalement connu et surtout peu protégé au titre des Monuments historiques, les rares protections étant tardives, légères et liées pour une grande part à la reconnaissance de leur concepteur plutôt qu'à leur place au sein de l'histoire de l'architecture scolaire ${ }^{58}$. Metz et ses lycées n'échappent pas à ce constat. Ainsi, bien que les lycées Fabert, Georges-de-La-Tour et Louis-Vincent semblent pleinement ancrés dans le patrimoine messin ${ }^{59}$, à l'exception de l'internat du lycée Fabert qui a obtenu en 2015 le label «Patrimoine du $\mathrm{xx}^{\mathrm{e}}$ siècle" (devenu depuis "Architecture contemporaine remarquable »), aucun établissement messin n'est labellisé ou protégé dans sa globalité au titre des Monuments historiques.

Plusieurs lycées bénéficient cependant d'une protection patrimoniale. Ainsi, le lycée Fabert se trouve-t-il en totalité dans le site des Thermes classé depuis 1927, ce qui implique un passage devant la commission départementale des Sites ainsi qu'un visa de la direction régionale de l'Environnement, de l'Aménagement et du Logement (Dreal) pour tous les travaux prévus dans ce lycée ou ses espaces extérieurs. À cela s'ajoute la proximité immédiate de nombreux monuments historiques: les baies de l'ancienne caserne du Cloître, dans la cour de l'internat (classées en 1926), mais aussi la basilique Saint-Vincent, le Temple neuf ou encore le théâtre (tous classés au titre des Monuments historiques en 1930). L'incidence de ce cadre réglementaire se fait tout particulièrement ressentir dans les années 1990, quand l'établissement souhaite se doter d'un espace de restauration. Six hypothèses sont soumises aux services de l'État, que le service départemental de l'Architecture et du Patrimoine se voit contraint de refuser. Une implantation à l'intérieur du lycée semblant alors inenvisageable, de 
nouvelles solutions sont recherchées sur des terrains situés à proximité, suscitant pendant plusieurs années de grands débats, des articles dans la presse, des passes d'armes entre conseil d'administration, associations, Ville, région, services de l'État, professeurs, étudiants, etc. Cette tension semble atteindre son paroxysme avec l'organisation d'une manifestation, en avril 1997, rassemblant un millier d'élèves, enseignants et sympathisants usés par un dossier qui commence à lasser tous les usagers de Fabert ${ }^{60}$. En parallèle, des concours architecturaux sont engagés puis abandonnés. C'est finalement en faisant appel à un architecte du patrimoine messin, Jacques Fabbri, qu'une solution est trouvée. Ce dernier mène une étude historique sur les bâtiments du lycée et retrouve la trace de l'ancien hôpital Saint-Georges, dont l'emprise proche du bâtiment des Sciences permettrait d'imaginer d'implanter tout de même la cantine dans les murs du lycée. Cette analyse historique convainc l'architecte des Bâtiments de France, qui accepte de laisser construire une partie de la cantine sur les fondations de cet ancien hôpital, demandant en contrepartie que la Région s'engage à budgétiser des travaux sur l'ensemble Fabert à chaque programme pluriannuel d'investissement sur les lycées. La construction de la cantine peut donc entrer dans une phase active ${ }^{61}$. Le rez-de-chaussée du bâtiment des Sciences étant réaffecté aux salles de restauration, les classes préparatoires sont alors contraintes, par un jeu de chaises musicales, de déménager, entraînant un début de réorganisation du site. Ainsi, en 1999, le lycée Fabert s'agrandit en intégrant le bâtiment « Tocqueville », un ancien immeuble d'habitation reconverti, adossé à l'internat, qui offre une quinzaine de salles destinées à accueillir les classes préparatoires. En 2003, les espaces de restauration de l'établissement sont enfin inaugurés ${ }^{62}$; d'autres travaux suivent, dont la réalisation du $\mathrm{CDI}^{63}$ en 2011. Précisons que le vieillissement des bâtiments du lycée exige des travaux réguliers, qui se poursuivent : après la restauration des façades de l'internat (2018-2020), des constructions modulaires temporaires ont été mises en place à l'automne 2020 pour accueillir les classes de l'ancien orphelinat Sainte-Constance dont l'état sanitaire va nécessiter des études de diagnostic et sans doute un chantier.

Le lycée Georges-de-La-Tour, place de Maud'Huy, est dans le périmètre de 500 mètres de plusieurs monuments historiques, dont l'église Sainte-Thérèse. Son annexe, située à proximité, au sein de la cité scolaire Barbot, doit-elle aussi respecter les contraintes liées à sa localisation, aux abords de nombreux monuments, tels le palais du Gouverneur (inscrit en 1975) ou encore l'hôtel des Arts et Métiers (inscrit en 2002). En 2009, l'agence KL architectes met en œuvre une restructuration lourde du bâtiment Biologie de l'annexe Barbot [fig. 12]. 
Figure 12

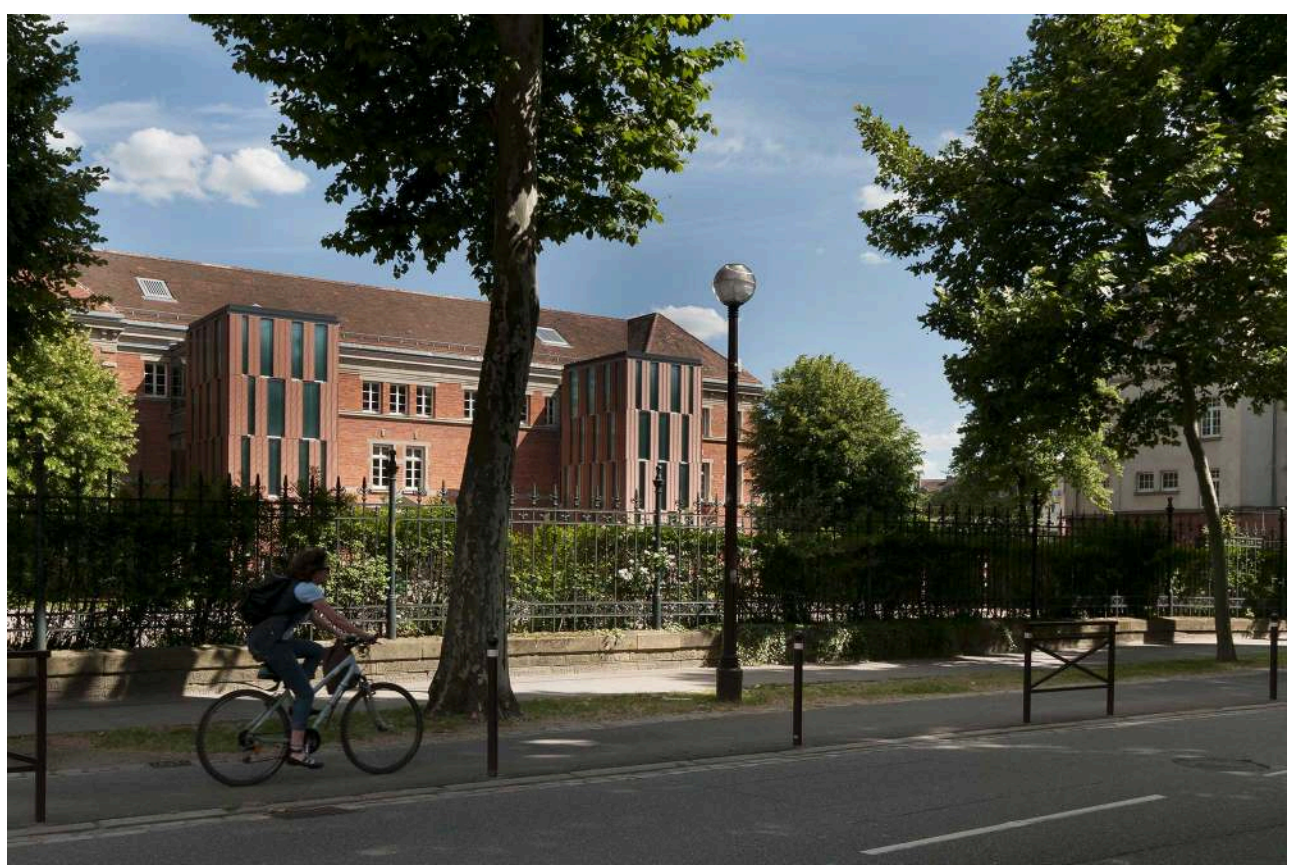

Le bâtiment Biologie de l'annexe Barbot du lycée Georges-de-La-Tour (cité scolaire Barbot) : intervention contemporaine de l'agence KL Architectes, 2009.

(c) KL Architectes.

La question des servitudes patrimoniales survient quand il faut déporter les cages d'escaliers à l'extérieur de l'existant plutôt que de les conserver dans un intérieur trop contraint. La création de ces escaliers extérieurs avec un jeu de peaux donne l'occasion à l'agence de poser un geste architectural fort et respectueux du site puisqu'elle prend le parti de rompre avec l'architecture existante par l'adjonction de volumes abstraits, qui reprennent néanmoins les couleurs de l'existant, offrant un dialogue réussi entre architecture ancienne et contemporaine ${ }^{64}$. Dix ans plus tard, l'agence La Como, associée à Jacques Fabbri pour ce qui est du volet patrimonial ${ }^{65}$, réhabilite quant à elle le bâtiment des Sciences du bâtiment principal, place de Maud'Huy.

Le site du lycée Louis-Vincent, exigu et situé dans aux abords de l'église Sainte-Thérèse de l'Enfant-Jésus (classée en 1998), ne permet plus désormais que des opérations de restructuration de l'existant. Quant au lycée Louis-de-Cormontaigne, il est situé dans le périmètre de l'église Saint-Simon-et-Saint-Jude et de la place de France (inscrites en 1989).

Depuis l'extension du secteur sauvegardé par l'arrêté préfectoral du 25 octobre 2010, le site patrimonial remarquable de Metz englobe le lycée Fabert et le lycée Georges-deLa-Tour. Cependant, aucun des autres lycées n'est situé dans un périmètre de protection patrimonial spécifique, même si une réflexion patrimoniale mériterait d'être menée sur cette thématique. En effet, la préservation des principaux témoins bâtis de l'histoire de l'enseignement à Metz apparaît nécessaire. Une réflexion sur la valeur patrimoniale des lycées ne bénéficiant d'aucune protection serait justifiée, en particulier pour le lycée Robert-Schuman, édifié pendant la période des Trente Glorieuses et témoin essentiel de l'histoire des lycées messins, et dont l'architecture doit pouvoir s'adapter aux enjeux contemporains de l'enseignement sans lui faire perdre son identité ${ }^{6}$. Il est enfin assez symptomatique d'observer que seuls les 
lycées Fabert et Louis-Vincent ouvrent leurs portes à l'occasion des Journées européennes du patrimoine, ce qui reflète ici encore le chemin qu'il reste à parcourir pour valoriser encore davantage le patrimoine des lycées à Metz.

Aujourd'hui, l'heure ne semble plus être aux nouveaux projets ex nihilo mais à l'adaptation à de nouvelles exigences, plus durables voire connectées, sous l'égide de la Région Lorraine puis Grand Est, depuis le $1^{\mathrm{er}}$ janvier 2016. Si le lycée Jean-Victor Poncelet a fermé ses portes en $2015^{67}$ faute de dispenser une formation suffisamment diversifiée, le lycée Robert-Schuman est passé de 4500 à 2000 élèves, ce qui pose nécessairement la question de son évolution et de son adaptation, de la même façon que les lycées Fabert et Georges-de-La-Tour, plébiscités, doivent continuer à se repenser. L'adaptation des lycées au monde contemporain semble engagée et ces lieux vivants se transforment pour répondre aux normes d'accessibilité et aux enjeux de développement durable et de respect de l'environnement, sujets qui pourront contribuer à ancrer ces constructions des deux derniers siècles dans le nôtre ${ }^{68}$.

\section{NOTES}

1. Synonyme de "territoire communal », cette expression est employée dans le Haut-Rhin, le Bas-Rhin et en Moselle.

2. CORDANI Aline, Le Lycée Fabert, 200 ans d'histoire, Metz, Serpenoise, 2006, p. 569-574, [disponible en ligne] https://journals.openedition.org/histoire-education/2094 [lien valide en mars 2021].

3. Notons que l'expression allemande "höhere Schule" ne correspond pas tout à fait aux établissements regroupés en France dans la catégorie " enseignement secondaire ». Elle désigne tant les établissements classiques (Gymnasium, Progymnasium, Lateinschule, mais aussi Lyceum ici) que les établissements modernes (Realschule, Oberrealschule ou encore Real Gymnasium). Voir: ROTH François, La Lorraine annexée. Étude sur la Présidence de Lorraine dans l'Empire allemand, 1870-1918, Metz, Serpenoise, 2007 [1976], p. 163.

4. ROTH François, La Lorraine annexée, p. 576.

5. Voir PIGNON-FELLER Christiane, Metz, 1848-1918. Les métamorphoses d'une ville, Paris, Éditions du Patrimoine, 2013.

6. En Allemagne, les écoles supérieures de filles existent depuis la fin du xviII ${ }^{\mathrm{e}}$ siècle. Pour davantage d'informations sur ces questions, voir ETTWILLER Éric, "L'Enseignement secondaire des filles en Alsace-Lorraine et dans l'académie de Nancy de 1871 à 1940 », Revue d'Alsace, n 143, "protestants et protestantisme en Alsace de 1517 à nos jours ", 2017. Pour un plus ample développement, voir la thèse de doctorat dont est issu cet article: ETTWILLER Éric, «L'Enseignement secondaire des filles en Alsace-Lorraine et dans l'académie de Nancy de 1871 à 1940 ", thèse de doctorat d'histoire sous la direction de Claude Muller, soutenue à l'université de Strasbourg (Strasbourg, 2017), [en ligne] https://journals.openedition.org/alsace/2608 [lien valide en mars 2021].

7. Né à Niederaula (province de Hesse-Nassau) en 1851, Conrad Wahn a étudié à la Technische Hochschule de Hanovre. Il est nommé à Metz en 1875 et devient architecte municipal en 1887.

8. Archives municipales de Metz (AMM), délibérations du conseil municipal de la Ville de Metz, 23 janvier 1906. 
9. SCHNEIDER Romana, "Tendances de l'architecture scolaire en Allemagne au xxesiècle", Histoire de l'éducation, $\mathrm{n}^{\circ}$ 102, "L'architecture scolaire», 2004 [en ligne], http:// journals.openedition.org/histoire-education/706 [lien valide en mars 2021].

10. AMM 4M101-110.

11. S. N., « Die Feiern zur Übersiedlung der städtischen Höheren Mädchenschule », Metzer Zeitung, 29 juillet 1910. Cette mention se trouve dans un article de presse publié à l'occasion de l'inauguration de l'école.

12. WILCKEN Niels, Metz et Guillaume II : architecture et pouvoir: l'architecture publique à Metz au temps de l'Empire allemand (1871-1918), Metz, Serpenoise, 2007, p. 69.

13. PIGNON-FELLER Christiane \& LEGAY Christian (photographe), Metz impérial. Patrimoine, intérieurs et art de vivre, 1871-1918, Ars-sur-Moselle, Serge Domini, 2011, p. 164-167.

14. Rapport explicatif du 20 juin 1911, cité par WILCKEN Niels, Metz et Guillaume II, p. 70.

15. MASSEL Christiane, MAURER Pierre \& PIGNON-FELLER Christiane, Metz au temps de l'Art déco. Urbanisme \& architecture, 1919-1939, Ars-sur-Moselle, Serge Domini, 2016, p. 83-93.

16. Une partie du cloître avait été ainsi divisée pour accueillir, depuis les premières années du lycée, diverses salles.

17. Cette peinture représente un panorama de la ville de Metz. Il est à noter que, durant l'entredeux-guerres, bien avant la création du $1 \%$ artistique, la Ville de Metz confiait à des artistes la création d'œuvres d'art destinées à des édifices construits ou gérés par la commune.

18. Deuxième Grand Prix de Rome en 1928.

19. CORDANI Aline, Le Lycée Fabert, 200 ans d'histoire, p. 548-549.

20. GROSDIDIER de MATONS Jean, Metz d'un petit garçon, Metz, Serpenoise, 1989, p. 73.

21. CHAMOUX Émile, L'Internat du lycée de Metz, Nancy, Imprimerie Helio-Lorraine, 1936.

22. «Lycée de garçons à Metz. Architecte : Parisot ", L'Architecture d'aujourd'hui, n 1, 1933, p. 91.

23. LAPRADE Albert, «L'Architecture dans nos provinces françaises : le lycée de Metz, R. Parisot et P. Millochau, architectes », L'Architecture, n 7, 1937, p. 227-238.

24. MARGERAND J.-L., « Internat du lycée de garçons de la ville de Metz par Robert Parisot \& Paul Millochau Architectes SADG », La Construction moderne, $\mathrm{n}^{\circ}$ 32, 1937, p. 696-705.

25. P. L., "Académie d'art national. Concours 1936 d'architecture régionale municipale », Art national construction, $\mathrm{n}^{\circ} 69$, 1937, p.109-111, [disponible en ligne] https://gallica.bnf.fr/ark:/ 12148/bpt6k55952532/f23.item.zoom [lien valide en mars 2021].

26. Pour prolonger, voir : MAURER Pierre, «Internat du lycée Fabert, Metz », in ETCHART MariePascale, GLOC Marie, PEZZOLI Carole, JUHEL Enola, MOHAUPT Pauline, PREZ Anne-Lise \& KEYNE Richard, Architecture du XXe siècle. Grand Est, Lyon, Lieux Dits, 2018, p. 14-17.

27. On peut citer ici en exemple le lycée Jules-Ferry à Paris (Pierre Paquet architecte, 1913), ancien lycée de jeunes filles, ou encore plus largement les constructions scolaires parisiennes de la III République et de l'entre-deux-guerres, qui emploient amplement la brique et sont publiés dans différentes revues d'architecture (La Construction moderne, L'Architecture, etc.).

28. MASSEL Christiane, MAURER Pierre \& PIGNON-FELLER Christiane, Metz au temps de l'Art déco..., p. 88-89.

29. Ibid., p. 83-87.

30. MONDON Raymond, GAILLOT Charles, WOLFF Robert, MEYER, HOCQUARD Camille et al., Dix ans de vie municipale 1947-1957, Metz, s. n., 1958, p. 44.

31. Jean Berthoin, dernier ministre de l'Éducation nationale de la IVe République (1958-1959).

32. AMM 1D/f2, délibérations du conseil municipal de la Ville de Metz, 2 août 1946, point 18.

33. CORDANI Aline, Le Lycée Fabert, 200 ans d'histoire, p. 608-610.

34. S. N., «D'importants travaux au lycée de garçons permettront de loger dignement les cours de sciences physiques ou naturelles », Le Républicain lorrain, 25 mai 1954. 
35. S. N., «Du nouveau au lycée de garçons. Le bâtiment des classes de géographie, dessin, sciences physiques et histoire naturelle sera ouvert pour la rentrée prochaine ", Le Républicain lorrain, 20 septembre 1956.

36. AMM 2PC12769 : lycée Fabert.

37. CORDANI Aline, Le Lycée Fabert, 200 ans d'histoire, p. 3.

38. Ibid., p. 384-385.

39. Ibid., p. 415.

40. S. N., "Les effectifs d'élèves ayant doublé depuis la guerre, le lycée de garçons de Metz ne peut plus suffire. La création d'un nouvel établissement est envisagée », Le Républicain lorrain, 28 janvier 1955.

41. AMM, délibérations du conseil municipal de la Ville de Metz, 29 décembre 1958, point 17.

42. AMM 36W10.

43. S. N., « 3000 élèves à la rentrée 1963 à Metz-Plantières où le lycée classique et moderne et le lycée technique commercial mixte sortent de terre ", Le Républicain lorrain, 18 février 1962.

44. S. N., "L'ancien lycée canadien "Général Navereau" abritera à la rentrée le collège littéraire universitaire de Metz », Le Républicain lorrain, 14 juillet 1968 (AMM 36W3).

45. S. N., "Le nouveau lycée prend forme en bordure de la route de Strasbourg ", Le Républicain lorrain, 23 avril 1963.

46. S. N., «L'inauguration du lycée R.-Schuman à Plantières et la visite ministérielle au collège scientifique de Saulcy », Le Républicain lorrain, 27 avril 1964.

47. S. N., "L'ouverture du nouveau lycée de Queuleu-Plantières entraînera en septembre une réorganisation scolaire à Metz ", Le Républicain lorrain, 11 mai 1963.

48. Cette réalisation, pour laquelle les sources primaires semblent en grande partie lacunaires, mériterait d'être développée dans le cadre d'autres recherches.

49. Voir sur ce sujet : MARCHAND Marie-Jacqueline, «Les lycées, les régions : an III », Politiques et management public, 1991, vol.9, n 1, p. 47-66, [disponible en ligne] https://www.persee.fr/doc/ pomap_0758-1726_1991_num_9_1_2975 [lien valide en mars 2021].

50. AMM 149W52: direction générale de l'Urbanisme et du Développement, Technopôle Metz 2000, lycée de la Communication, 31 mars 1988.

51. S. N., « Un parc d'activités technologiques à Metz », L'Est républicain, 21 mai 1984.

52. AMM 6PC70 : AAG sarl, Alpha architecture, SCPA, «Mémoire. Lycée de la Communication. Note architecturale », vers 1988.

53. M. G., « Lycée du commerce et de la distribution. Un nouveau tonus pour le quartier de MetzNord ", Le Républicain lorrain, 10 décembre 1991.

54. AMM 284W108.

55. AMM 284W107.

56. JAGER Jean-Pierre, "Lycée du Commerce à Metz. Chemin faisant le bois épouse le béton », Le Républicain lorrain, 10 octobre 1993.

57. AMM 284W107: «La région de Lorraine. Ville de Metz. Concours du lycée régional du commerce et de la distribution. Avant-projet sommaire définitif », avril 1992.

58. CHÂTELET Anne-Marie, "L'architecture scolaire", in CONDETTE Jean-François \& FIGEACMONTHUS Marguerite (dir.), Sur les traces du passé de l'éducation... Patrimoines et territoires de la recherche en éducation dans l'espace français, Pessac, Maison des sciences de l'homme d'Aquitaine, coll. « Épistémologie », 2014, p. 55-65, [disponible en ligne] https://books.openedition.org/msha/ 597?lang=fr [lien valide en mars 2021].

59. Tous bénéficient de publications spécifiques, plus ou moins denses, qui mettent en avant leur intérêt historique voire architectural.

60. CORDANI Aline, Le Lycée Fabert, 200 ans d'histoire, p. 627-646.

61. Maîtrise d'œuvre : Denu et Paradon Architectes, Strasbourg.

62. CORDANI Aline, Le Lycée Fabert, 200 ans d'histoire, p. 622-627. 
63. Maîtrise d'œuvre : La Como, Strasbourg.

64. Pour en savoir plus : https://kl-architectes.fr/projets/restructuration-du-batiment-biologieau-lycee-georges-de-la-tour-de-metz/ [lien valide en mars 2021].

65. Pour les édifices sur des sites historiques, le groupement d'architectes doit contenir au moins un architecte du patrimoine.

66. Il y a près de trente ans, les services de la Région se sont demandé s'il était plus intéressant de raser entièrement le lycée pour tout reconstruire ou s'il valait mieux le conserver. D'après le témoignage de Mario Fardelli (recueilli le 9 décembre 2020), les larges couloirs et les proportions généreuses des différents bâtiments ont plaidé contre le projet de démolition.

67. CALVEZ Gaël, «Fermeture du lycée Poncelet à Metz: après le choc, la résignation", Le Républicain Lorrain, 10 avril 2015.

68. L'auteur tient à remercier tout spécialement Mario Fardelli, responsable du pôle Construction et Maintenance (agence territoriale de Metz) à la Région Grand-Est, pour le temps qu'il a bien voulu lui consacrer. Merci également à Catherine Guégan pour sa relecture attentive et enfin à Anne-Marie Châtelet, Sandrine Cocca, Agnès Lechleiter, Christiane Massel, Olivier Mathiotte et Christiane Pignon-Feller pour leur aide précieuse.

\section{RÉSUMÉS}

Nous tenterons dans cet article d'analyser les dynamiques d'implantation, de création et d'extension des lycées à travers le cas d'une ville de province, Metz (Moselle), depuis le lycée impérial jusqu'aux lycées actuels en passant par ceux construits durant l'annexion de la ville à l'Allemagne (1871-1919), les extensions remarquables de l'entre-deux-guerres et enfin les constructions industrialisées des Trente Glorieuses. Cette étude transversale d'édifices publics sur un territoire communal défini s'inscrit inévitablement dans une mise en perspective entre les aspects locaux et nationaux, comme le choix des architectes qui sont à l'origine de ces constructions. Nous distinguerons les faits saillants de l'histoire de ces édifices, sur près de deux siècles, en mettant en exergue le contexte de leur construction et leur évolution.

This article takes as a case study a provincial city in an attempt to analyse the dynamics of location, creation and extension of the high schools of Metz, from the imperial high school to the current high schools, including those built during the annexation of the city to Germany (1871-1919), the remarkable extensions of the inter-war period, and finally the industrialised constructions of the "Trente Glorieuses", the 30 years of post-war economic boom in France. This transversal reading of public buildings within a defined communal territory inevitably fits into a perspective between the local and the national, for example in the choice of the architects behind these constructions. Covering nearly two centuries, this article highlights the history of these buildings, emphasising the context of their construction and their evolution. 
INDEX

Keywords : France, Metz, Lorraine (region), lycée Fabert in Metz, lycée Georges-de-La-Tour in Metz, lycée Louis-Vincent in Metz, lycée Robert-Schuman in Metz, lycée de la Communication in Metz, lycée René-Cassin in Metz

Mots-clés : Metz, Lorraine, lycée Fabert à Metz, lycée Georges-de-La-Tour à Metz, lycée LouisVincent à Metz, lycée Robert-Schuman à Metz, lycée de la Communication à Metz, lycée RenéCassin à Metz

\section{AUTEUR}

\section{PIERRE MAURER}

Docteur en histoire de l'architecture, École d'architecture de Nancy, LHAC pierre.maurer@nancy.archi.fr 